\title{
The expression of selected molecular markers of immune tolerance in psoriatic patients
}

\author{
Joanna Bartosińska ${ }^{1, A-E}$, Joanna Purkot ${ }^{2, B, C}$, Małgorzata Kowal ${ }^{1, B}$, Anna Michalak-Stoma ${ }^{1, B}$, \\ Dorota Krasowska ${ }^{1, E, F}$, Grażyna Chodorowska ${ }^{1, A, C, E, F}$, Krzysztof Giannopoulos ${ }^{2, A, C, E, F}$ \\ ${ }^{1}$ Department of Dermatology, Venereology and Pediatric Dermatology, Medical University of Lublin, Poland \\ 2 Department of Experimental Hematooncology, Medical University of Lublin, Poland \\ A - research concept and design; $B$ - collection and/or assembly of data; $C$ - data analysis and interpretation; \\ $\mathrm{D}$ - writing the article; $\mathrm{E}$ - critical revision of the article; $\mathrm{F}$ - final approval of the article
}

Address for correspondence

Joanna Bartosińska

E-mail: jbartosinski@gmail.com

Funding sources

The study was supported by DS164 of the Medical University of Lublin.

The publication fee was covered by DS462/2016

of the Medical University of Lublin.

Conflict of interest

None declared

Received on July 14, 2017

Reviewed on July 31, 2017

Accepted on September 26, 2017

\begin{abstract}
Background. Psoriasis is a chronic autoinflammatory disease whose underlying molecular mechanisms remain unclear. The disease is mediated by the cells and molecules of both the innate and adaptive immune systems. Some T cell surface molecules, including neuropilin-1 (NRP1), programmed death 1 (PD-1) and the human leukocyte antigen $G(H L A-G)$, are known to play a role in the maintenance of immune tolerance.

Objectives. The aim of this study was to investigate HLA-G, NRP1 and programmed cell death gene (PDCD1) mRNA expression in psoriatic patients.

Material and methods. The study included 72 psoriatic patients and 35 healthy individuals. Twentyone patients (29.17\%) suffered from concomitant psoriatic arthritis. The mRNA expression of HLA-G, NRP1, and PDCD1 were determined using quantitative real-time reverse transcription polymerase chain reaction (qRT-PCR). The severity of skin lesions was assessed by means of the Psoriasis Area and Severity Index (PASI), Body Surface Area (BSA), the Patient Global Assessment (PGA), and the Dermatology Life Quality Index (DLQI).

Results. The median value of the PASI was 11.5, and of BSA was 15.8\%. The expressions of NRP1 and PDCD1, but not HLA-G, were significantly lower in psoriatic patients in comparison with the control group. The expression of HLA-G, NRP1 and PDCD1 were not significantly different in the psoriatic arthritis and psoriasis vulgaris patients.
\end{abstract}

Conclusions. The results of this study suggest that the molecular markers of immune tolerance, i.e., HLA-G, NRP1, and PD-1, may be involved in the immune response in psoriatic patients.

Key words: psoriasis, PD-1, HLA-G, NRP1

DOI

10.17219/acem/78020

\section{Copyright}

Copyright by Author(s)

This is an article distributed under the terms of the

Creative Commons Attribution Non-Commercial License

(http://creativecommons.org/licenses/by-nc-nd/4.0/) 


\section{Introduction}

A disruption to the immune system is likely to compromise immune tolerance and may lead to the development of autoimmune and autoinflammatory conditions.

In psoriasis, whose pathogenesis involves keratinocyte hyperplasia and excessive angiogenesis, the role of genetic factors, environmental triggers and aberrant immune system regulation resulting in compromised immune tolerance is well understood. Here, the $\mathrm{T}$ cell immune response is activated by antigen-presenting cells (APCs) which will undoubtedly impair the balance between the effector and regulatory components of the immune system. ${ }^{1}$

Physiologically, in order to maintain undisturbed immune tolerance, various cellular and molecular mechanisms become activated. These include the central negative selection of autoreactive $\mathrm{T}$ cells in the thymus as well as peripheral anergy, clonal deletion, suppression, and exhaustion. This natural immune tolerance depends on the interaction between the T cells' stimulatory and inhibitory receptors and their ligands. ${ }^{2}$ All of them form a complex network of molecules that interact in order to maintain peripheral tolerance or to enhance $\mathrm{T}$ cell immunity.

Since certain T cell surface molecules, including neuropilin-1 (NRP1), programmed death 1 (PD-1), and human leukocyte antigen G (HLA-G) play a vital role in the T cells' interactions with APCs (including dendritic cells [DCs], B cells, monocytes and macrophages), their compromised suppressive ability could insufficiently inhibit or even fail to inhibit the activity of the inflammatory cells in psoriasis.

Neuropilin-1, a transmembrane glycoprotein and a receptor involved in some physiological and pathological processes (including angiogenesis), acts as a co-receptor for the vascular endothelial growth factor (VEGF) and semaphorins 3A (Sema 3A). ${ }^{3}$ Neuropilin- 1 is preferentially expressed on regulatory $\mathrm{T}$ cells (Tregs) and myeloid cells (i.e., DCs), and it promotes long-term interactions between Tregs and immature DCs, thereby suppressing their activation and function. ${ }^{4}$ A decreased number of NRP1-positive Treg cells, however, could possibly explain the inability of the immune system to suppress an immune response and restore immune tolerance.

Programmed death 1, a glycoprotein expressed on T cells - including Tregs - B cells, monocytes, and macrophages, is a known checkpoint inhibitor which blocks the effector T cells' function and promotes Treg activity. The co-expression of the PD-1 with its ligand 1 (PD-L1) on Tregs enhances their proliferation and favors the maintenance of self-tolerance. ${ }^{5}$

Human leukocyte antigen G, a nonclassical human leukocyte antigen (HLA), known as a tolerogenic molecule, is capable of downregulating the natural killer (NK) cells' cytotoxic activity, the inhibition of $\mathrm{T}$ cell migration, the induction of the apoptosis of activated CD8+ T cells, and the prevention of DC maturation. It induces the functional silencing of the immune response, which is indispensable in certain physiological settings, e.g., during pregnancy, but it also allows the tumor cells to escape the host's immune system. ${ }^{6}$ This leukocyte affects the antigen presentation by the inhibition of HLA II upregulation and its interaction with co-stimulatory molecules. ${ }^{7}$

The 3 aforementioned molecules, i.e., HLA-G, NRP1 and PD-1, need to be better understood in terms of their function in psoriasis. Since all the molecular markers are capable of inhibiting the $\mathrm{T}$ cell function through the action on both innate and adaptive immunity, it is worth studying whether they are differently expressed in psoriatic patients compared to healthy individuals. If the coexistence of abnormal immunomodulatory properties of HLA-G, an absence of PD-1 co-inhibitory signal and a lack of NRP1 positive $\mathrm{T}$ cells with suppressive ability proved to be of significance in psoriasis, then chronic autoinflammatory disease, self-reactivity and the persistent T-cell activation in psoriasis could be better understood.

\section{Material and methods}

\section{Study group}

The study comprised 72 psoriatic patients, including 21 (29.17\%) psoriatic arthritis patients, hospitalized in the Department of Dermatology, Venereology, and Pediatric Dermatology at the Medical University of Lublin, Poland, as well as 35 age- and gender-matched healthy volunteers.

The study inclusion criteria were a duration of psoriasis of at least 1 year, the presence of active psoriatic skin lesions, and an age of at least 18 years. The exclusion criteria were cardiovascular, cerebrovascular, hematologic, hepatic, or renal disease; neoplasm; chronic viral infections; erythrodermic, pustular, or guttate psoriasis; addiction to drugs; and systemic anti-psoriatic treatment.

The study was approved by the Local Ethics Committee at the Medical University of Lublin (KE-0254/81/2015). Informed consent was obtained from all participants.

\section{Assessment of psoriasis severity}

The severity of psoriatic skin lesions was assessed with the Psoriasis Area and Severity Index (PASI), Body Surface Area (BSA), Patient Global Assessment (PGA), the Dermatology Life Quality Index (the DLQI), and nail plate changes with the Nail Psoriasis Severity Index 80 (NAPSI 80). Psoriatic arthritis (PsA) was diagnosed using the Classification of Psoriasis Arthritis criteria (CASPAR). The severity of psoriatic arthritis was assessed with the Disease Activity Score 28 (DAS 28), the Patient Visual Analog Scale (VAS), the number of tender joints, the number of swollen joints, and a physician-patient Likert scale.

\section{Assessment of gene expression}

NRP1, PDCD1 and HLA-G mRNA expression was measured by quantitative real-time reverse transcription-polymerase chain reaction (qRT-PCR). 
First, peripheral blood mononuclear cells (PBMCs) from psoriatic patients and healthy volunteers were isolated by Ficoll density gradient centrifugation (Biochrom AG, Berlin, Germany) and cryopreserved at $-80^{\circ} \mathrm{C}$ until the time of analysis. The viability of the PBMCs obtained was always $>95 \%$, as determined by Trypan blue staining. Viable cells were quantified in a Neubauer chamber (Zeiss, Oberkochen, Germany).

Next, total RNA was isolated from the PBMCs using a QIAamp RNA Blood Mini Kit (Qiagen, Venlo, Netherlands) and reverse-transcribed to cDNA using a QuantiTect Reverse Transcription Kit (Qiagen). cDNA was used in a qRT-PCR to measure the mRNA expression of NRP1 (Hs00826128_m1), PDCD1 (Hs0155088_m1) and HLA-G (Hs00365950_g1) using the TaqMan Gene Expression Assay methodology, according to the manufacturer's protocol (Applied Biosystems, Foster City, USA). Glyceraldehyde3-phosphate dehydrogenase (GAPDH) was used as a constitutively expressed housekeeping gene and negative controls contained water instead of cDNA to ensure the purity of all reagents. The thermocycling program was set for 40 cycles of $15 \mathrm{~s}$ at $95^{\circ} \mathrm{C}$ and $1 \mathrm{~min}$ at $60^{\circ} \mathrm{C}$ on the ABI Prism 7300 Sequence Detector (Applied Biosystems).

The NRP1, PDCD1, and HLA-G mRNA expression was calculated as inverse ratios of the differences in the cycles of threshold $\left(1 / \Delta C_{t}\right)$, where $\Delta C_{t}$ is the $C_{t}$ value of the target receptors minus the $\mathrm{C}_{\mathrm{t}}$ value of $G A P D H$.

\section{Statistical analysis}

The results were statistically analyzed with STATISTICA v. 10.0 PL (StatSoft Inc., Tulsa, USA). The figures were created using the GraphPad Prism 5 software (GraphPad Software Inc., San Diego, USA). Median values with minimal and maximal values were estimated for continuous variables, or absolute (n) and relative numbers (\%) of the occurrence of items for categorical variables. Mann-Whitney U test was used to compare the mRNA expressions of NRP1, $H L A-G$, and $P D C D 1$ between the psoriatic patients and the control group, as well as between the psoriatic patients with and without arthritis. Spearman's rank correlation coefficient was used to investigate the correlation between the mRNA expressions of NRP1, HLA-G and PDCD1, and the clinical features of psoriasis and psoriatic arthritis. A value of $\mathrm{p}<0.05$ was considered to be significant.

\section{Results}

Most of the patients were male, with an average age of 47 years and an average psoriasis duration of 15.5 years. The severity of psoriasis assessed by the PASI was 11.5 , by BSA 15.8 , and by the DLQI 12.0, on average. Psoriatic arthritis was present in the studied patients for 6 years on average. More than half of the PsA patients had the oligoarticular clinical subset of the disease (Table 1).
The psoriatic patients had a significantly lower mRNA expression of NRP1 (median: 0.09) in comparison to the healthy controls (median: 0.14). Similarly, the psoriatic patients had a significantly lower mRNA expression of PDCD1 (median: 0.08) in comparison to the healthy controls (median: 0.11). The mRNA expression of $H L A-G$ did not significantly differ between the psoriatic patients (median: 0.05) and the healthy controls (median: 0.05) (Fig. 1).

None of the studied molecular markers of immune tolerance significantly differed between the psoriatic arthritis and psoriasis vulgaris patients (Fig. 2). The median mRNA expression of NRP1 was 0.09 , for PDCD1 it was 0.08 , and for HLA-G it was 0.05 for both psoriatic arthritis and psoriasis vulgaris patients.

The studied mRNA expression of HLA-G correlated negatively only with the age of the psoriatic patients. However, the studied mRNA expressions of PDCD1 and NRP1 did not correlate with any clinical features either in the psoriatic or the psoriatic arthritis patients (Table 2).

Table 1. Clinical data of psoriatic patients

\begin{tabular}{|c|c|c|}
\hline Patients & Clinical data & Estimate \\
\hline \multirow{12}{*}{$\begin{array}{l}\text { Psoriasis } \\
(n=72)\end{array}$} & age [years], median (min-max) & $47(21-76)$ \\
\hline & female (n) & 11 \\
\hline & male (n) & 61 \\
\hline & $\begin{array}{l}\text { duration of the disease [years], median } \\
\qquad(\min -\max )\end{array}$ & $15.5(1-55)$ \\
\hline & $\begin{array}{l}\text { age at disease onset [years], median } \\
\qquad(\min -\max )\end{array}$ & $22(1-71)$ \\
\hline & positive family history (n, \%) & $27(37.50)$ \\
\hline & PASI, median (min-max) & $11.5(3-49)$ \\
\hline & PGA, median (min-max) & $3(2-5)$ \\
\hline & BSA (\%), median (min-max) & $15.8(2-75)$ \\
\hline & DLQI, median (min-max) & $12(1-30)$ \\
\hline & NAPSI, median (min-max) & $14(0-64)$ \\
\hline & diagnosis of psoriatic arthritis (n, \%) & $21(29.17)$ \\
\hline \multirow{8}{*}{$\begin{array}{l}\text { Psoriatic } \\
\text { arthritis } \\
(\text { PsA) } \\
(n=21)\end{array}$} & $\begin{array}{l}\text { duration of psoriatic arthritis [years], median } \\
\text { (min-max) }\end{array}$ & $6(1-20)$ \\
\hline & number of tender joints, median (min-max) & $5(0-12)$ \\
\hline & number of swollen joints, median (min-max) & $0(0-6)$ \\
\hline & physician Likert, median (min-max) & $2(1-4)$ \\
\hline & patient Likert, median (min-max) & $3(1-4)$ \\
\hline & DAS28, median (min-max) & $3.4(1.0-5.5)$ \\
\hline & VAS, median (min-max) & $50(0-70)$ \\
\hline & $\begin{array}{c}\text { clinical subsets of PsA (n) } \\
\text { oligoarticular }(\leq 5 \text { joints }) \\
\text { polyarticular } \\
\text { distal interphalangeal predominant } \\
\text { spondylitis predominant } \\
\text { mutilans }\end{array}$ & $\begin{array}{c}13(61.90 \%) \\
6(28.57 \%) \\
2(9.53 \%) \\
0 \\
0\end{array}$ \\
\hline
\end{tabular}

PASI - Psoriasis Area and Severity Index; PGA - Patient Global Assessment; BSA - Body Surface Area; DLQI - Dermatology Life Quality Index; NAPSI - Nail Psoriasis Severity Index; DAS 28 - Disease Activity Score; VAS - Patient Visual Analog Scale. 


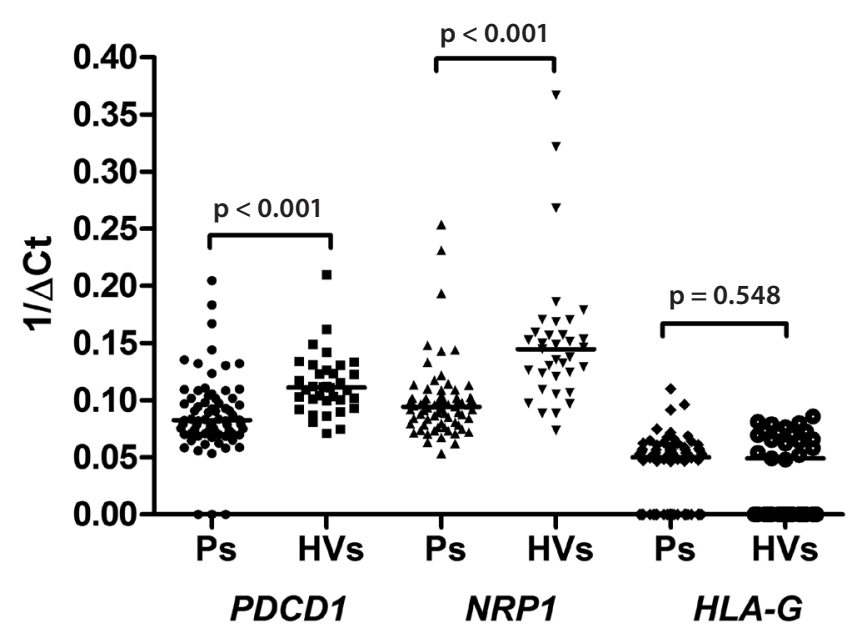

Fig. 1. Comparison of median PDCD1, NRP1, and HLA-G mRNA expression between psoriatic patients (Ps) and healthy volunteers (HVs)

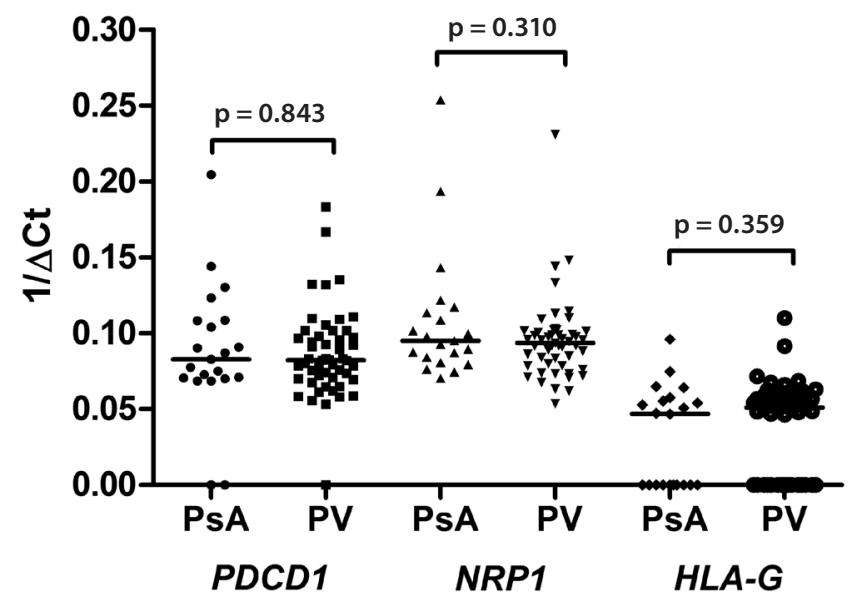

Fig. 2. Comparison of median PDCD1, NRP1, and HLA-G mRNA expression between psoriatic arthritis (PSA) and psoriasis vulgaris (PV) patients

Table 2. Correlation coefficients between the studied molecular markers and clinical data of psoriatic patients

\begin{tabular}{|c|c|c|c|c|}
\hline Patients & Clinical data & PDCD1 & NRP1 & $H L A-G$ \\
\hline \multirow{8}{*}{$\begin{array}{l}\text { Psoriasis } \\
(n=72)\end{array}$} & age [years] & -0.08 & -0.06 & $-0.36^{*}$ \\
\hline & duration of the disease [years] & -0.10 & 0.00 & -0.19 \\
\hline & age at disease onset [years] & 0.02 & -0.08 & -0.09 \\
\hline & PASI & -0.09 & -0.10 & 0.10 \\
\hline & PGA & -0.04 & -0.09 & 0.03 \\
\hline & BSA (\%) & -0.07 & -0.11 & 0.06 \\
\hline & DLQI & -0.08 & -0.11 & -0.04 \\
\hline & NAPSI & -0.13 & -0.14 & -0.07 \\
\hline \multirow{6}{*}{$\begin{array}{l}\text { Psoriatic } \\
\text { arthritis } \\
(P s A) \\
(n=21)\end{array}$} & duration of PsA [years] & -0.29 & -0.18 & -0.14 \\
\hline & age of PsA onset [years] & -0.05 & -0.29 & -0.40 \\
\hline & number of tender joints & -0.19 & -0.18 & -0.13 \\
\hline & number of swollen joints & -0.16 & -0.14 & 0.01 \\
\hline & DAS28 & -0.17 & -0.09 & 0.16 \\
\hline & VAS & -0.05 & -0.09 & -0.09 \\
\hline
\end{tabular}

* $p<0.05 ;$ PASI - Psoriasis Area and Severity Index; PGA - Patient Global Assessment; BSA - Body Surface Area; DLQI - Dermatology Life Quality Index; NAPSI - Nail Psoriasis Severity Index; DAS 28 - Disease Activity Score; VAS - Patient Visual Analog Scale.

\section{Discussion}

Any imbalance between the regulatory $\mathrm{T}$ cells (Tregs) and the effector T cells - including Th1, Th17, and Th22 - is pivotal in triggering psoriasis, while a subset of CD4+ T cells - Tregs with the CD25+Foxp-3+ (Forkhead box p3) phenotype - is known to prevent autoimmunity and to maintain immunological homeostasis and peripheral immune tolerance. ${ }^{8}$ Nevertheless, under special circumstances, e.g., in inflammatory processes, the Tregs may undergo conversion into effector T cells (Th1 or Th17). ${ }^{9}$ The number and function of Tregs are known to be downregulated in both the peripheral blood and skin lesions of psoriatic patients. The efficient inhibition of Th1 and Th17 activity requires the presence of interleukin 10 (IL-10) and transforming growth factor $\beta$ (TGF- $\beta$ ), the major anti-inflammatory cytokines produced by various cells of the immune system, including Tregs. ${ }^{10}$ Therefore, abnormally low levels of regulatory cytokines or their divergent action in inflammatory conditions - also observed in psoriasis - are not able to balance the enhanced Th1/Th17 response in this disease. ${ }^{11}$

Apart from the CD25+Foxp-3+ Tregs, HLA-G+ Tregs that emerge from the thymus also suppress $\mathrm{T}$ cell proliferation. Moreover, the HLA-G-negative APCs are able to induce the activation and proliferation of the T cells, while the HLAG-positive APCs induce the differentiation of the Tregs. ${ }^{12}$

Interleukin 10 is the main upregulator of HLA-G expression, a molecule that may promote the development of the Tregs. However, not only anti-inflammatory cytokines, but also some growth factors and proinflammatory cytokines - such as IL-1 $\beta$, IFN- $\alpha$, IFN- $\beta$, IFN- $\gamma$, GM-CSF, and EGF - are capable of upregulating the $H L A-G$ gene expression. ${ }^{13}$

In our study, no difference was found in the mRNA expression of $H L A-G$ between the studied psoriatic patients and the healthy volunteers.

The research conducted so far has been focused on comparing the HLA-G protein levels in the psoriatic plaque and healthy skin.

In their immunohistochemical studies of psoriatic skin, Aractingi et al. found the presence of the HLA-G protein expression in all of the examined sections of the skin, which was not detected in the normal skin. ${ }^{14}$ Since the HLA-G in Aractingi et al. study was mainly expressed by macrophages, the authors concluded that they could be representative of a control system which might be capable of counteracting the auto-reactive $\mathrm{T}$ cells. ${ }^{14}$

Similarly, Cardili et al. revealed an increased HLA-G expression in psoriatic skin in comparison with the skin of healthy individuals. ${ }^{15}$ Sweeney C and Kirby B suggested that in psoriasis the HLA-G may promote the development of Tregs and prevent tissue destruction. ${ }^{16}$

Bearing in mind the results obtained by Aractingi and other researchers, as well as the results of our study, the presence of some inhibitory feedback mechanism powerful enough to downregulate the deleterious effect of the $\mathrm{T}$ cells and to prevent destruction in psoriatic skin is highly probable. 
The analysis of NRP1 expression in psoriatic epidermis conducted by some authors has yielded contradictory results. Although some of them have reported an increased expression of NRP1 in psoriatic lesions, others have shown opposite results. Interestingly, in our study we observed a decreased mRNA expression of NRP1 in the psoriatic patients in comparison to the healthy controls, which may confirm its role in disrupting immune tolerance in psoriatic patients.

Furthermore, Kou et al., in both immunostaining and mRNA expression studies, demonstrated lower NRP1 and Sema3A in psoriatic skin samples compared with healthy skin samples. ${ }^{17}$ Henno et al., however, showed an increased expression of not only NRP1 but also other pro-angiogenic factors, i.e., VEGF-A, VEGFR2, and PIGF, in psoriatic lesions compared to the uninvolved skin of the studied psoriatic patients. ${ }^{18}$ The same authors also observed a significant overexpression of NRP1 in the nonlesional psoriatic skin in comparison to the skin of healthy volunteers.

The decreased NRP1 expression detected in our study may suggest the presence of some specific mechanisms of NRP1 regulation in peripheral blood, different from those in psoriatic plaques. The role of NRP1 as a co-receptor for VEGFR2 is important in the skin for blood vascular network expansion.

As in the case of PD-1, studies on its expression in psoriasis are scarce and performed on small series cases, they present contradictory results. Nevertheless, there are some reports pointing to the induction of psoriasis or a psoriasislike disease during treatment with anti-PD-1 agents (i.e., nivolumab, pembrolizumab and pidilizumab). ${ }^{19}$ This is not unusual since PD-1 is a molecule capable of suppressing $\mathrm{T}$ cell activity and favoring Treg proliferation. Therefore, its blockage will provoke a shift of cellular reactivity towards the pro-inflammatory Th1/Th17 lymphocytes.

Kim et al., in their quantitative real-time RT-PCR, western blotting, and immunohistochemistry studies have found a decreased expression of PD-1 ligands (PD-L1 and PD-L2) in the psoriatic epidermis in comparison to the healthy controls. ${ }^{20}$ They suggested that it could result from an impairment of the Tregs' function in psoriasis and that it could allow continuous $\mathrm{T}$ cell activation.

Kim et al., in their animal study on imiquimod-treated mice, demonstrated overexpressed PD-1 on IL-17A-producing $\mathrm{T}$ cells. ${ }^{21}$ The same authors observed similar results in the skin of psoriatic patients in whom immunofluorescent staining also revealed an overexpression of PD-1 on IL-17A-producing T cells.

Contrary to the studies conducted so far, which focused on the PD-1 expression in the skin, our present investigation provides some insight into the mRNA PDCD1 expression. In our study, mRNA PDCD1 expression has proven to be lower in the psoriatic patients, which is in agreement with its mechanisms of immune response. One possible limitation of our study may be the number of patients in the psoriatic arthritis group.
The study results for the molecular markers of immune tolerance, i.e., HLA-G, NRP1, and PDCD1, conducted so far give reasonable grounds for making further efforts to broaden scientific knowledge about the possible mechanisms involved in the induction and maintenance of the immune response in psoriatic patients.

\section{References}

1. Deng $Y$, Chang $C, L u$ Q. The inflammatory response in psoriasis: A comprehensive review. Clin Rev Allergy Immunol.2016;50(3):377-389.

2. Bardhan K, Anagnostou T, Boussiotis VA. The PD1:PD-L1/2 pathway from discovery to clinical implementation. Front Immunol. 2016;12(7): 550. doi: 10.3389/fimmu.2016.00550

3. Oussa NA, Dahmani A, Gomis M, et al. VEGF requires the receptor NRP-1 to inhibit lipopolysaccharide-dependent dendritic cell maturation. J Immunol. 2016;197(10):3927-3935.

4. Sarris M, Andersen KG, Randow F, Mayr L, Betz AG. Neuropilin-1 expression on regulatory $T$ cells enhances their interactions with dendritic cells during antigen recognition. Immunity. 2008;28(3):402-413.

5. Chen Z, Pang N, Du R, et al. Elevated expression of programmed death-1 and programmed death ligand-1 negatively regulates immune response against cervical cancer cells. Mediators Inflamm. 2016;2016:6891482.

6. Urosevic M. HLA-G in the skin - friend or foe? Semin Cancer Biol. 2007;17(6):480-484.

7. Amodio G, Sales de Albuquerque R, Gregori S. New insights into HLA-G mediated tolerance. Tissue Antigens. 2014;84(3):255-263.

8. Wang Y, Wang L, Yang H, Yuan W, Ren J, Bai Y. Activated circulating T follicular helper cells are associated with disease severity in patients with psoriasis. J Immunol Res. 2016;2016:7346030.

9. Smilek DE, Ehlers MR, Nepom GT. Restoring the balance: Immunotherapeutic combinations for autoimmune disease. Dis Model Mech. 2014;7(5):503-513.

10. Borghi A, Fogli E, Stignani M, et al. Soluble human leukocyte antigen$\mathrm{G}$ and interleukin-10 levels in plasma of psoriatic patients: Preliminary study on a possible correlation between generalized immune status, treatments and disease. Arch Dermatol Res. 2008;300(10):551-559.

11. Han G, Li F, Singh TP, Wolf $P$, Wang $X-J$. The pro-inflammatory role of TGFß1: A paradox? Int J Biol Sci. 2012;8(2):228-235.

12. Brenol CV, Veit TD, Chies JA, Xavier RM. The role of the HLA-G gene and molecule on the clinical expression of rheumatologic diseases. Rev Bras Reumatol. 2012;52(1):82-91.

13. Moreau P, Flajollet $S$, Carosella ED. Non-classical transcriptional regulation of HLA-G: An update. J Cell Mol Med. 2009;13(9B):2973-2989.

14. Aractingi $S$, Briand N, Le Danff $C$, et al. HLA-G and NK receptor are expressed in psoriatic skin: A possible pathway for regulating infiltrating T cells? Am J Pathol. 2001;159(1):71-77.

15. Cardili RN, Alves TG, Freitas JC, et al. Expression of human leucocyte antigen-G primarily targets affected skin of patients with psoriasis. Br J Dermatol. 2010;163(4):769-775.

16. Sweeney C, Kirby B. Does HLA-G prevent tissue destruction in psoriasis? Br J Dermatol. 2011;164(5):1118-1119.

17. Kou K, Nakamura F, Aihara M, et al. Decreased expression of semaphorin-3A, a neurite-collapsing factor, is associated with itch in psoriatic skin. Acta Derm Venereol. 2012;92(5):521-528.

18. Henno A, Blacher S, Lambert CA, et al. Histological and transcriptional study of angiogenesis and lymphangiogenesis in uninvolved skin, acute pinpoint lesions and established psoriasis plaques: An approach of vascular development chronology in psoriasis. JDermatol Sci. 2010;57(3):162-169.

19. Ruiz-Bañobre J, García-González J. Anti-PD-1/PD-L1-induced psoriasis from an oncological perspective. J Eur Acad Dermatol Venereol. Epub 2017 Apr 3. doi: 10.1111/jdv.14217

20. Kim DS, Je JH, Kim SH, et al. Programmed death-ligand 1, 2 expressions are decreased in the psoriatic epidermis. Arch Dermatol Res. 2015;307(6):531-538.

21. Kim JH, Choi YJ, Lee BH, et al. Programmed cell death ligand 1 alleviates psoriatic inflammation by suppressing IL-17A production from programmed cell death 1-high T cells. J Allergy Clin Immunol. 2016; 137(5):1466-1476. 